LA W RENCE LIVERMORE N A TION AL LABORATORY
Interpreting Mammalian Evolution using Fugu Genome Comparisons

I. Ovcharenko, L. Stubbs, G. G. Loots

April 7, 2004

Genomics 
This document was prepared as an account of work sponsored by an agency of the United States Government. Neither the United States Government nor the University of California nor any of their employees, makes any warranty, express or implied, or assumes any legal liability or responsibility for the accuracy, completeness, or usefulness of any information, apparatus, product, or process disclosed, or represents that its use would not infringe privately owned rights. Reference herein to any specific commercial product, process, or service by trade name, trademark, manufacturer, or otherwise, does not necessarily constitute or imply its endorsement, recommendation, or favoring by the United States Government or the University of California. The views and opinions of authors expressed herein do not necessarily state or reflect those of the United States Government or the University of California, and shall not be used for advertising or product endorsement purposes. 


\section{Interpreting Mammalian Evolution using Fugu Genome Comparisons}

Ivan Ovcharenko*, Lisa Stubbs and Gabriela G. Loots*

Genome Biology Department

Lawrence Livermore National Laboratory

7000 East Ave, L-441

Livermore, CA 94550

Fax: 925-422-2099

*correspondence should be addressed to:

Ivan Ovcharenko

Tel: (925) 422-5035

Email: ovcharenko1@llnl.gov

or

Gabriela G. Loots

Tel: 925-423-0923

Email: loots1@llnl.gov. 
Comparative sequence analysis of the human and the pufferfish Fugu rubripes (fugu) genomes has revealed several novel functional coding and noncoding regions in the human genome $[1,2]$. In particular, the fugu genome has been extremely valuable for identifying transcriptional regulatory elements in human loci harboring unusually high levels of evolutionary conservation to rodent genomes [3-5]. In such regions, the large evolutionary distance between human and fishes provides an additional filter through which functional noncoding elements can be detected with high efficiency.

We have evaluated the noncoding conservation profile in human/fugu genome alignments obtained from the ECR Browser (http://ecrbrowser.dcode.org/) [6] and generated by the blastz program [7]. Filtering of known and putative transcripts, pseudogenes, GenBank mRNAs, as well as proximal promoter sequences identified 2,968 human/fugu evolutionary conserved regions (ECRs) $[\geq 70 \%$ identity (\%ID) over $\geq 100$ basepairs (bp)] that are noncoding in nature and distantly positioned from the transcriptional start sites of adjacent genes. These ECRs are predominantly clustered in discrete areas of the human genome, flanked by or inserted into the introns of 1,026 human transcripts that together comprise only $5.6 \%$ of the 18,410 'known Gene' loci (as annotated at UCSC Genome Browser [8], hg16 freeze). This distribution suggests that human-fugu sequence comparisons will be beneficial for identifying noncoding regulatory elements for only a small percentage of human genes. Moreover, the number of genes under the control of these putative regulatory elements could be even smaller if enhancers located between two genes influence gene expression of only one of the neighboring transcripts. 
It has been estimated that $\sim 5 \%$ of a mammalian genome is under active selection, the majority of which will likely correspond to functional coding and noncoding sequences [9]. Human-rodent genome alignments [6] revealed 1.3 million noncoding ECRs with an average distribution of 68.8 ECRs per human gene locus, whereas the density varies according to the regional neutral substitution rates [10]. Assigning in vivo function to all these conserved elements is impossible with current technologies, and it is therefore critically important to identify ways to efficiently discriminate functional noncoding elements from neutrally evolving, but still highly conserved genomic DNA. This goal might be achieved if 'fingerprints' unique to functional and non-functional noncoding conserved elements can be defined. Assuming that elements conserved between human and fugu represent an incomplete yet highly enriched functional dataset, we approached this problem by studying signatures specific to human/mouse conserved noncoding elements that are also present in fishes.

We analyzed the distribution in size and percent identity of human/rodent $(\mathrm{h} / \mathrm{r})$ and human/fugu $(\mathrm{h} / \mathrm{f})$ noncoding ECRs, and compared these datasets with the $\mathrm{h} / \mathrm{r}$ ECRs that are also conserved in fugu (Figure 1). Under represented regions in the mouse genome were extended by the available rat genomic sequences, in order to create a comprehensive $\mathrm{h} / \mathrm{r}$ ECR dataset. The distribution in ECR length was strikingly similar between the human/mouse and human/fugu ECRs comparisons; $81 \% \mathrm{~h} / \mathrm{r}$ and $86 \% \mathrm{~h} / \mathrm{f}$ ECRs were shorter than 350 basepairs (bp). In sharp contrast, the majority of h/r ECRs that are conserved in fugu (90\% ID) were greater than 350 bps in length. Similar striking differences were observed for the level of sequence identity. While $82 \%$ and $71 \%$ of the $\mathrm{h} / \mathrm{m}$ and $\mathrm{h} / \mathrm{f}$ ECRs were found to range between $70 \%$ and $77 \%$ sequence identity, $90 \%$ of 
$\mathrm{h} / \mathrm{m}$ ECRs also conserved in fugu showed greater than $77 \%$ level of sequence identity. Therefore, our analysis suggests that a "mammalian evolutionary threshold" of $\geq 350 \mathrm{bps}$, $\geq 77 \%$ ID conservation criteria recapitulates the majority of all conserved noncoding elements identified from $\mathrm{h} / \mathrm{f}$ genome comparisons, and reduces the number of $\mathrm{h} / \mathrm{m}$ conserved noncoding elements 10 -fold, from 1.3 millions to 128,000 ECRs, significantly simplifying the search for putative functional noncoding elements.

To correlate our findings with the conservation profiles of known regulatory elements we analyzed a $2.6 \mathrm{Mb}$ region from the human $D A C H$ gene locus where recently seven human enhancers have been mapped [5]. Of the $1367 \mathrm{~h} / \mathrm{r}$ noncoding ECRs (>100 $\mathrm{bp} />70 \%$ ID), 34 are also present in fugu. A conservation criteria of $\geq 350 \mathrm{bp} / \geq 77 \%$ ID identifies $302 \mathrm{~h} / \mathrm{r}$ ECRs and recapitulates 33/34 of the $\mathrm{h} / \mathrm{f}$ conserved elements while it excludes $78 \%$ of the original $\mathrm{h} / \mathrm{m}$ ECRs and maintains $100 \%$ of the experimentally validated regulatory elements [5]. Other known distant regulatory elements, including SHH and DLX1 specific developmental enhancers exceed this conservation threshold ( $\geq 350 \mathrm{bp} / \geq 77 \%$ ID) in $\mathrm{h} / \mathrm{r}$ genomic alignments, independent of their presence in the fugu genome (Table 1) [11, 12]. We also applied these newly defined parameters on humanchicken and human-frog whole genome alignments available at the ECR Browser [6]. Over $72 \%$ of $\sim 7500$ human-frog and $55.4 \%$ of $\sim 71,200$ human-chicken noncoding ECRs that are also present in rodents obey the "mammalian evolutionary threshold" rule of conservation in the analysis of human-rodent counterpart ECRs. As we move closer in evolution within the vertebrate radiation, the significant decrease in the ratio of depicted ECRs provides a magnifying glass that allows us to visualize functional regions lacking sufficient evolutionary time to diverge in neutral regions. 
Concluding, we suggest a novel approach for analyzing human/rodent conservation profiles that is capable of reconstructing more ancestral evolutionary relationships and distinguishing functional conserved elements from the neutrally evolving genomic background. By applying a ' $>350 \mathrm{bps} />77 \%$ ' threshold to the analysis of human/rodent conservation profiles we were able to recapitulate the majority of human/fish conserved elements and to generate a small set of elements that have a high probability of being functional noncoding domains. Similar statistical approaches will be critical to understanding phylogenetic relationships through systematic pair-wise genomic comparisons, and has the potential to facilitate the identification of regulatory elements specific to recently evolved species such as humans and their primate relatives.

Acknowledgements: This work was supported by LLNL-LDRD 04-ERD-052. The work was performed under the auspices of the U.S. Department of Energy by the University of California, Lawrence Livermore National Laboratory Contract No. W-7405-Eng-48. 
Table 1. Experimentally characterized distant enhancer elements in the mouse.

\begin{tabular}{|c|c|c|c|c|}
\hline ECR-gene & Enhancer & Size $(\mathrm{h} / \mathrm{m})$ & H/M \%ID & fugu cons \\
\hline Dachhund & Nobrega MA et al. 2003 & & & \\
\hline Dc1 & negative & 630 & \multicolumn{2}{|c|}{$89 \% \mathrm{y}$} \\
\hline Dc2 & hindbrain & 1405 & \multicolumn{2}{|c|}{$89 \% \mid y$} \\
\hline Dc3 & for-, hind-brain spinal cord, retina & 2458 & \multicolumn{2}{|c|}{$88 \% \mathrm{y}$} \\
\hline Dc4 & Retina & 1132 & \multicolumn{2}{|c|}{$83 \% y$} \\
\hline Dc5 & negative & 730 & \multicolumn{2}{|c|}{$88 \% \mathrm{y}$} \\
\hline Dc6 & midbrain, redina, drg & 891 & \multicolumn{2}{|c|}{$89 \% y$} \\
\hline Dc7 & limb bud & 1401 & \multicolumn{2}{|c|}{$88 \% y$} \\
\hline Dc8 & forbrain, neural tube & 1023 & \multicolumn{2}{|c|}{$87 \% y$} \\
\hline Dc9 & Hindbrain, neural tube, genitalia & 2247 & \multicolumn{2}{|c|}{$82 \% y$} \\
\hline DIx1-2 & Ghanem $\mathrm{N}$ et al. 2003 & & & \\
\hline $112 a$ & mesenchyme cells, branchyal arch & 1784 & \multicolumn{2}{|c|}{$84 \% \mid y$} \\
\hline $112 b$ & telencephalon, diencephalon & 864 & \multicolumn{2}{|c|}{$92 \% \mid y$} \\
\hline DIx5-6 & Ghanem $\mathrm{N}$ et al. 2003 & & & \\
\hline $\mathrm{ml56i}$ & telencephalon & 1477 & \multicolumn{2}{|c|}{$88 \% \mid y$} \\
\hline ml56ii & forbrain & 830 & \multicolumn{2}{|c|}{$88 \% \mid y$} \\
\hline SHH & Lettice LA et al. 2003 & 1205 & \multicolumn{2}{|c|}{$83 \% y$} \\
\hline Hoxc8 & Anand S et al. 2003 & 583 & \multicolumn{2}{|c|}{$82 \% y$} \\
\hline IL4/IL13 & Loots GG et al. 2000 & 472 & \multicolumn{2}{|c|}{$79 \%$ no } \\
\hline FGF4 & Luster TA et al. 2003 & 566 & \multicolumn{2}{|c|}{$81 \%$ no } \\
\hline pax6/nkx2.8 & Fabio Santagati et al 2003 & & & \\
\hline cns6 & & 500 & \multicolumn{2}{|c|}{$83 \% \mid y$} \\
\hline cns+2 & & 1600 & \multicolumn{2}{|c|}{$82 \% y$} \\
\hline $\operatorname{pax} 7$ & Deborah Lang et a. 2003 & & & \\
\hline intron1 & & 608 & $85 \%$ & no \\
\hline ApoE & Zheng et al. 2004 & & & \\
\hline brain & & 420 & $75 \% n$ & no \\
\hline
\end{tabular}


Figure 1. Genome scan of ECR length (A) and percent identity (B). Human/fugu ECRs are in blue, human/rodent ECRs are in orange, and human/rodent ECRs conserved to fugu are in green. $x$-axis, size in bp (A) and percent identity (B); $y$-axis, number of ECRs.

A

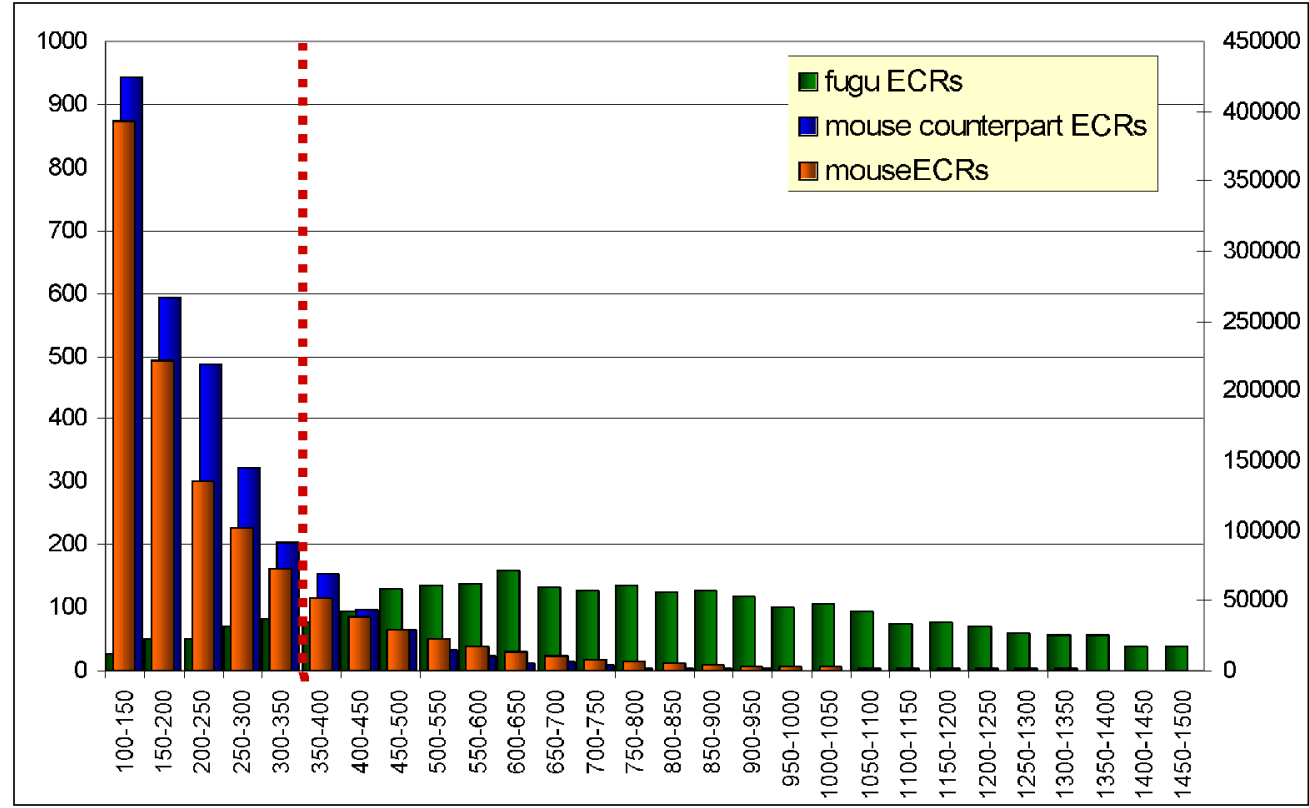

B

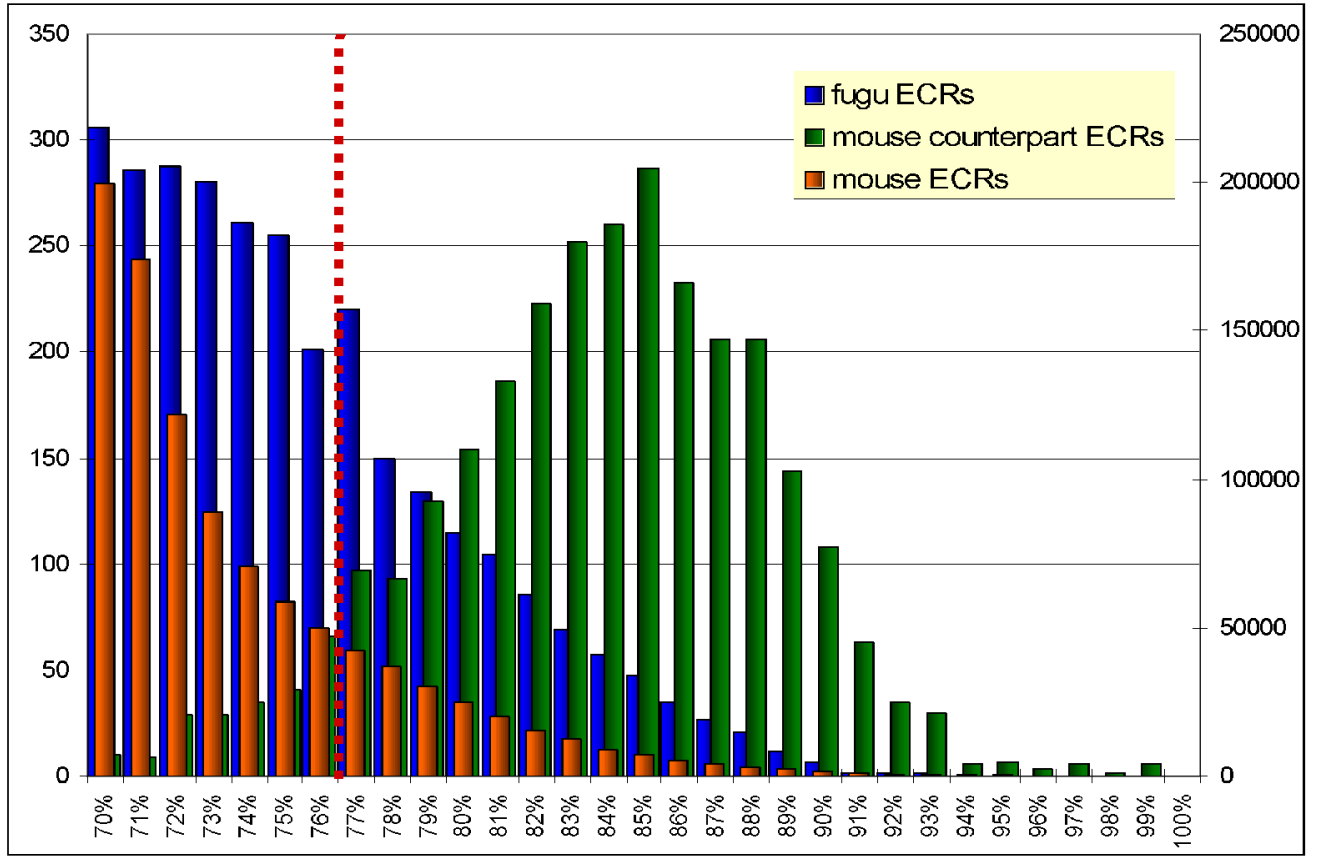




\section{References:}

1. Aparicio, S., et al., Whole-genome shotgun assembly and analysis of the genome of Fugu rubripes. Science, 2002. 297(5585): p. 1301-10.

2. Venkatesh, B., P. Gilligan, and S. Brenner, Fugu: a compact vertebrate reference genome. FEBS Lett, 2000. 476(1-2): p. 3-7.

3. Santagati, F., et al., Identification of Cis-regulatory elements in the mouse Pax9/Nkx2-9 genomic region: implication for evolutionary conserved synteny. Genetics, 2003. 165(1): p. 235-42.

4. Spitz, F., F. Gonzalez, and D. Duboule, A global control region defines a chromosomal regulatory landscape containing the HoxD cluster. Cell, 2003. 113(3): p. 405-17.

5. Nobrega, M.A., et al., Scanning human gene deserts for long-range enhancers. Science, 2003. 302(5644): p. 413.

6. Ovcharenko, I., Nobrega, M.A., Loots, G.G., and Stubbs, L., ECR Browser: A Tool For Visualizing And Accessing Data From Comparisons Of Multiple Vertebrate Genomes. Nucleic Acid Research, in press 2004.

7. Schwartz S, Kent WJ, Smit A, Zhang Z, Baertsch R, Hardison RC, Haussler D, Miller W. Human-mouse alignments with BLASTZ. (2003). Genome Res. 13:103-7.

8. Kent, W.J., Sugnet, C. W., Furey, T. S., Roskin, K.M., Pringle, T. H., Zahler, A. M., and Haussler, D. The Human Genome Browser at UCSC. (2002). Genome Res. 12:996-1006. 
9. Margulies, E.H., et al., Identification and characterization of multi-species conserved sequences. Genome Res, 2003. 13(12): p. 2507-18.

10. Hardison, R.C., et al., Covariation in frequencies of substitution, deletion, transposition, and recombination during eutherian evolution. Genome Res, 2003. 13(1): p. 13-26.

11. Lettice, L.A., et al., A long-range Shh enhancer regulates expression in the developing limb and fin and is associated with preaxial polydactyly. Hum Mol Genet, 2003. 12(14): p. 1725-35.

12. Ghanem, N., et al., Regulatory roles of conserved intergenic domains in vertebrate Dlx bigene clusters. Genome Res, 2003. 13(4): p. 533-43. 